PIT06 - Perception and Interactive Technologies, Irsee June 19-21 06, published in : Lecture Notes in Computer Science / Artificial Intelligence LNAI 4021 Springer, Heidelberg, Berlin (2006) pp.20-31

\title{
Modelling and Simulation of Spontaneous Perception Switching with Ambiguous Visual Stimuli in Augmented Vision Systems
}

\author{
Norbert Fürstenau \\ German Aerospace Center, Institute for Flight Guidance, Lilienthalplatz 7 \\ D-38108 Braunschweig, Germany \\ norbert.fuerstenau@dlr.de
}

\begin{abstract}
A behavioral nonlinear dynamics model of multistable perception due to ambiguous visual stimuli is presented. The perception state is formalized as the dynamic phase variable $\mathrm{v}(\mathrm{t})$ of a recursive process with cosinuidal transfer characteristic which is created by superposition (interference) of neuronal mean fields. The two parameters $\mu=$ difference of meaning of alternative percepts and $\mathrm{G}=$ attention parameter, control the transition between unambiguous and ambiguous stimuli, e.g. from stimulus off to stimulus on, and attention fatigue respectively. Mean field interference with delayed phase feedback enables transitions between chaotic and limit cycle attractors $v(t)$ representing the perception states. Perceptual reversals are induced by attention fatigue $\mathrm{G}(\mathrm{t})(\sim$ adaptive gain $g(v))$ with time constant $\gamma$, and attention bias which determines the relative duration of the percepts. The coupled attention - perception dynamics with an additive stochastic noise term reproduces the experimentally observed $\Gamma$-distribution of the reversal time statistics. Mean reversal times of typically 3 $-5 \mathrm{~s}$ as reported in the literature, are correctly predicted if delay $\mathrm{T}$ is associated with the delay of $40 \mathrm{~ms}$ between stimulus onset and primary visual cortex (V1) response. Numerically determined perceptual transition times of $3-5 \mathrm{~T}$ are in reasonable agreement with stimulus - conscious perception delay of $150-200$ $\mathrm{ms}$ [11]. Eigenfrequencies of the limit cycle oscillations are in the range of 10 $-100 \mathrm{~Hz}$, in agreement with typical EEG frequencies.
\end{abstract}

\section{Introduction}

From the use of (transparent) monocular head mounted displays (HMD) in military helicopters it is well known that different visual input into both eyes may involve adverse perceptual and attentional effects like binocular rivalry which may lead to significant reduction of reaction times [1]. Laramee et.al. [2] determined more than $100 \%$ response time increase in a HMD based visual search task due to binocular rivalry and visual interference effects. Binocular rivalry is the spontaneous involuntary switching of conscious awareness between the different percepts corresponding to the different stimuli of both eyes [3]. It belongs to a larger class of cognitive multistability effects as observed with ambiguous stimuli such as perspective reversal (e.g. the Necker cube [4][5]) or figure-ground reversal. Also dynamic stimuli may give rise to 
cognitive multistability, e.g. ambiguous motion displays such as plaids as induced by moving groups of crossed lines [6]. By determining the correlation dimension, e.g. of corresponding time series, Richards et.al [7] found experimental evidence indicating common nonlinear dynamical cognitive processes underlying a surprisingly diverse range of visual phenomena such as cognitive multistability with ambiguous pictures, saccade intervals in visual search, and movie scene durations. Binocular rivalry, however appears to be a predominantly stochastic phenomenon, in agreement with other authors [10][28][29].

The present macroscopic model provides an approach for explaining the experimental finding that deterministic (even chaotic) as well as stochastic dynamics determines the measured reversal time statistics for different multistability phenomena [7]. It contributes to the ongoing controversial discussion on the deterministic [8] [9] versus purely stochastic character [10] of cognitive multistability. In agreement with the widely accepted view of recursive interactions between distant neural groups leading to conscious perception (e.g. [11]), the model assumes a reentrant process which appears to be related to the dynamical core hypothesis of Tononi et.al. [12]. The model relies on the mean field phase oscillator theory of coupled neuronal columns in the visual cortex [13]. The latter was used for describing the synchronization of neuronal oscillations as the physiological basis of dynamic temporal binding which in turn is thought to be cruical for the selection of perceptually or behaviorally relevant information [14][15][16][18]. Self oscillation of neuronal groups within columns and coupling between columns is excited when the external stimulus exceeds a certain threshold [13]. Single columns exhibit multistable characteristics of the neuronal mean field as function of the stimulus, similar to the present model. Within the phase synchronization theory phase locking between different groups of neurons is described by means of the circle ( $\sin )$ map. Phase oscillator dynamics is the basis of the phase attractive circle map [17] which was used for describing human coordination dynamics as well as multistable perception.

A multistability model of Ditzinger \& Haken [8] is based on the continuous polynomial dynamics of two separate coupled perception state equations. In accordance with the experimentally supported satiation (neuronal fatigue) hypothesis [4] spontaneous transitions between different percepts are induced by the time variation of two attention (control) parameters due to perception - attention coupling. Recently published experimental results of Nakatani et.al.[19] support the perception - attention coupling approach. The present model follows the perception - attention coupling and attention fatigue approach in [8]. It takes into account, however, the reentrant character of the neuronal processes [12] by including a finite delay time $\mathrm{T}$, which results in limit cycle and chaotic attractor states defined as "percepts". In contrast to [8] a single differential - delay perception state equation together with a attention fatigue equation is formalized via the phase dynamics [13][17] of a recursive cosinuidal map as originating from superposition (interference) of neuronal mean fields. The stimulus ambiguity is quantified by a difference-of-meaning parameter which controls the stimuluson/-off switching. The present model provides an explicit quantitative confirmation of the proposed catastrophe topology of the cognitive multistability dynamics [20].

In the following section 2 I describe the theoretical approach, with details of the recursive interference model in subsection 2.1, and an analysis of the stationary behavior in section 2.2. Results of computer experiments are presented in section 3, with 
simulated time series and attention - perception phase space plots in subsection 3.1 and a statistical analysis of the reversal time intervals in section 3.2 with different reentrant delay times. The results are discussed with respect to published experimental data and alternative theoretical models in section 4. A conclusion and outlook is presented in section 5 .

\section{Theory}

\subsection{The Recursive Mean Field Interference Model}

As a kind of minimum architecture allowing for the emergence of discontinuous state transitions, I have proposed in previous papers coupling of the attention and perception dynamics via delayed phase feedback interference, and attention satiation[21][22]. Formally this is achieved analogous to multistable optical systems [23][24]. Interference with contrast $\mu$ is the superposition of (electromagnetic) fields $a_{0 i} \exp \left\{j\left(\omega t+\Phi_{i}\right)\right\}, \mathrm{i}=1,2,3, \ldots$ with $\omega=$ circular frequency, $\Phi=$ phase, and $\mathrm{a}_{0}=$ amplitude. The superposition yields extinction or amplification of each other, depending on the relative phase shift $\Delta \Phi=\Phi_{1}-\Phi_{2}$. It may be compared with the phase shift between the coupled self - oscillating neuronal columns of the mean field theory [13]. A simplified block diagram is depicted in Figure 1.

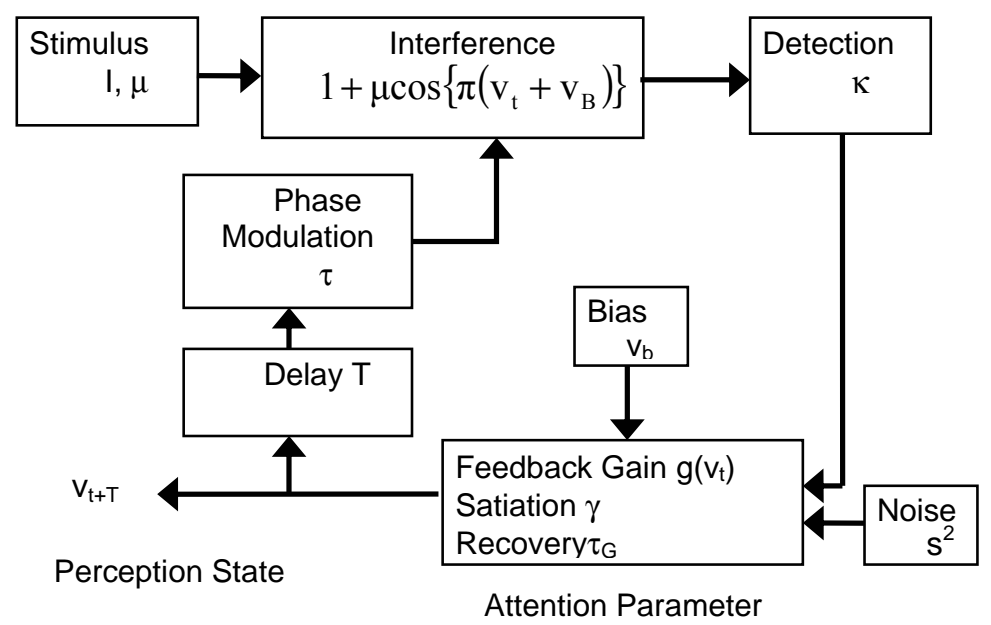

Fig. 1. Simplified block diagram of perception - attention recursive interference model. Symbols explained in the text

The feedback loop describing the perception state order parameter dynamics $v(t)$ may be compared with the reentrant thalamo - cortical and basal ganglia dynamics presented by Edelman [25] within his dynamical core hypothesis of consciousness: the interference and feedback gain blocks represent the thalamo - cortical complex 
and the sub - cortical basal ganglia with attention related tasks respectively. In the schematic an ambiguous stimulus with strength I and difference of meaning $\mu$ (= interference contrast $0 \leq \mu \leq 1$ ) of the two possible percepts $\mathrm{P} 1, \mathrm{P} 2$, excites two corresponding hypothetical mean fields with phase difference $\Delta \Phi=\pi \mathrm{v}_{\mathrm{t}}$. Interference contrast $\mu$ depends on the coherence and polarization of the two fields. It creates the typical cosinuidal dependence of the output (= squared modulus of the sum of the field amplitudes, with detection conversion factor $\kappa$ ) on the phase difference $\Delta \Phi$ as mapping function. A recurrent process is established by feedback of the output after amplification (feedback gain $g$, attention bias $v_{b}$, satiation (fatigue) and recovery time constants $\gamma$ and $\tau_{\mathrm{G}}$ ) with low pass filtering (time constant $\tau$ ) and delay T into $\Delta \Phi$ via a phase modulation mechanism. As a quantitative estimate for $\mathrm{T} I$ chose the stimulus primary visual cortex response delay $(\approx 40 \mathrm{~ms},[11])$. One possibility for phase feedback is frequency modulation of the input field [24], comparable to the stimulus induced modulation of the neuronal mean field limit cycle oscillations [13]. The normalized output $v_{t}=U_{t} / U_{\pi}$ of the feedback interference circuit with $d \Phi / d U=\pi / U_{\pi}$ and $U \sim$ percept intensity $=\mid$ superimposed percept field strength $\left.\right|^{2}$ defines the perception state $\mathrm{v}$ as synergetic order parameter. The phase attractive circle map as proposed by Kelso et.al. [9][17] represents a similar recursive (discrete) phase oscillator mapping function which, however attempts to model multistability without feedback gain as control parameter and insofar cannot be mapped to the reentrant loops as proposed by Edelman [25].

According to Hillyard et.al. [26] stimulus-evoked neuronal activity can be modified by an attentional induced additive bias or by a true gain modulation (present model parameters $v_{b}, G(t) \sim g$ ). Increase of gain is correlated with increased blood flow through the respective cortical areas. Recent experimental evidence on perception attention coupling with ambiguous stimuli was presented by Nakatani \& van Leeuven [19] using EEG recording of frontal theta and occipital alpha bands and eye blink rate measurement. Accordingly in the present model, like in [8], the feedback gain serves as adaptive control parameter ( attention parameter $G$ ) which induces the quasi - discontinuous transitions between the alternative stationary perception states $\mathrm{P} 1$ and $\mathrm{P} 2$, through attention satiation or fatigue [4]. A strongly damped (overdamped) feedback system is assumed with time constant $\tau>>$ coefficient of $\mathrm{d}^{2} \mathrm{v} / \mathrm{dt}^{2}$ which is neglected. Formally the model is described by coupling a nonlinear 1st order differential delay equation for $\mathrm{v}(\mathrm{t})$ with a linear equation for the control parameter dynamics $\mathrm{G}(\mathrm{t})$. In a first approach to model the unavoidable random disturbances due to dissipative processes, a stochastic force $\mathrm{L}(\mathrm{t})$ with Gaussian white noise (variance $\mathrm{s}^{2}$ ) is added to the attention equation $\mathrm{G}(\mathrm{t})$, similar to[8].

$$
\begin{gathered}
\tau \dot{\mathrm{v}}_{\mathrm{t}+\mathrm{T}}+\mathrm{v}_{\mathrm{t}+\mathrm{T}}=\mathrm{G}\left[1+\mu \cos \left(\pi\left(\mathrm{v}_{\mathrm{t}}+\mathrm{v}_{\mathrm{B}}\right)\right)\right] . \\
\dot{\mathrm{G}}_{\mathrm{t}}=\left(\mathrm{v}_{\mathrm{b}}-\mathrm{v}_{\mathrm{t}}\right) / \gamma+\left(\mathrm{G}_{\text {off }}-\mathrm{G}_{\mathrm{t}}\right) / \tau_{\mathrm{G}}+\mathrm{L}_{\mathrm{t}} .
\end{gathered}
$$

The rhs. of equ. (1a) describes the conventional interference between two coherent fields. In what follows I assume the phase bias $v_{B}=0 \bmod 2$. The attention parameter $\mathrm{G}(\mathrm{t})=\kappa \mathrm{I} g(\mathrm{t}) / \mathrm{U}_{\pi}$ with phase - voltage modulation factor $\mathrm{d} \Phi / \mathrm{dU}=\pi / \mathrm{U}_{\pi}$ is the prod- 
uct of feedback gain $\mathrm{g}(\mathrm{t})$ and input (stimulus strength) I (=1 in what follows). The attention dynamics is determined by attention bias $\mathrm{v}_{\mathrm{b}}$ (determining the relative preference of P1 and P2), satiation speed $1 / \gamma$, recovery time constant $\tau_{\mathrm{G}}$ and $\mathrm{G}_{\mathrm{off}}=$ attention (gain) parameter for stimulus off, defined by $\mu=\mu_{\text {off }}<0.18$ (see below).

The detailed block diagram of the model in Fig. 2 represents the highest hierarchy of an implementation in the graphical programming dynamical systems tool MatlabSimulink:

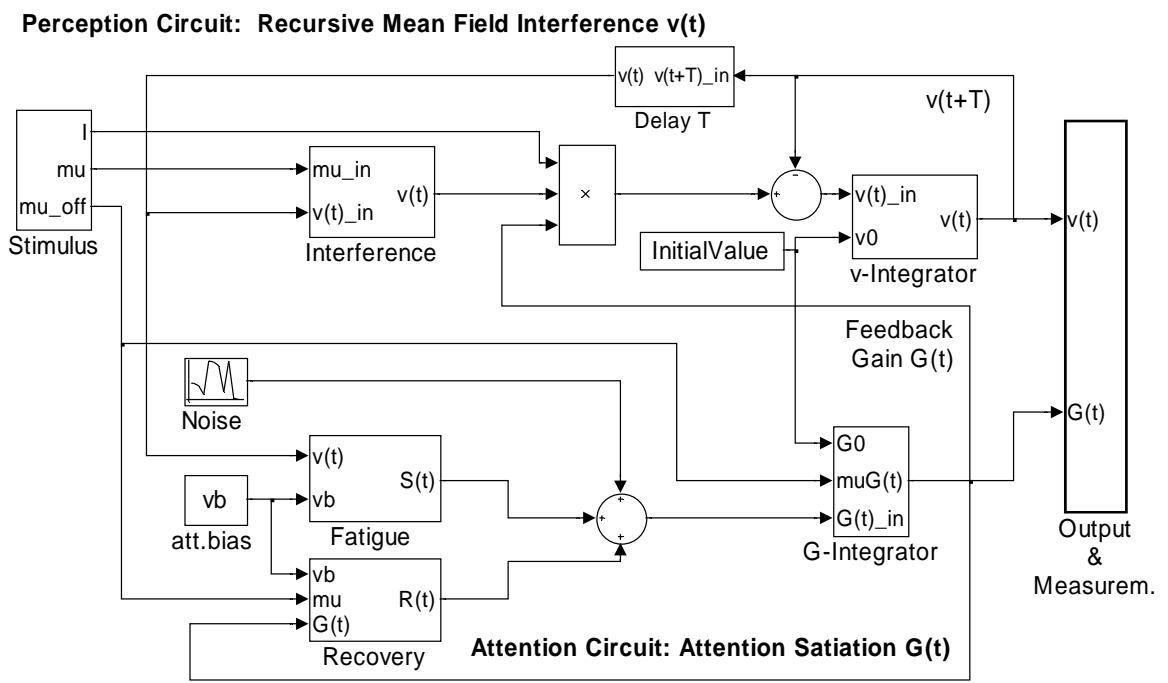

Fig. 2. Matlab - Simulink implementation (highest hierarchy level) of recursive interference model displaying subroutines (blocks) of the reentrant loops of perception circuit $v(t)$ in the upper half and attention circuit $G(t)$ in the lower half of the diagram. Stimulus of strength I and difference of meaning $m u(=\mu)$ are fed into perception circuit as control parameters with interference term and integrator loop with time constant $\tau$. Attention circuit $G(t)$ with satiation term (fatigue) $(v b-v(t)) / \gamma$ and recovery term $(G o f f-G(t)) / \tau_{G}$ controls as gain factor the perception dynamics. $\mathrm{v}(\mathrm{t})$ and $\mathrm{G}(\mathrm{t})$ output into the data evaluation block at the right

\subsection{Stationary Solutions of the Recursive Interference Equation}

Two types of instabilities are observed with recursive systems described by equation (1): period doubling and node bifurcation. Figure 3 depicts the stationary solutions $(\mathrm{dv} / \mathrm{dt}=0)$ including period doubling up to period $8, \mathrm{v}_{\mathrm{t}+\mathrm{iT}}=\mathrm{v}_{\mathrm{t}}=\mathrm{v}^{*}, \mathrm{i}=1,2,4,8$.

Period doubling pitchfork bifurcations are observed on both positive slope regions. The graph yields the control parameter values at the first three bifurcation points providing a first approximation to the Feigenbaum constant $\delta_{\infty}=4.6692$ via $\delta_{\infty}^{1} \approx\left(G_{2}-G_{1}\right) /\left(G_{3}-G_{2}\right)$. The period doubling behavior proves that within certain parameter ranges $(\mu, \tau)$ any system noise has chaotic contributions. This is confirmed by numerical evaluation of the Lyapunov coefficient [22]. 
In [21] I have shown the stationary solution of $v^{*}(\mu, G)$ to exhibit a topology similar to a cusp catastrophe. This finding agrees with a proposal of Poston \& Stewart [20] who developed a qualitative deterministic model of cognitive bistability based on catastrophe theory.

At the critical value, $\mu_{\mathrm{n}}=0.18$, node bifurcation is observed and the slope of the stationary system state $v^{*}$ as function of $G$ becomes infinite. For $\mu<\mu_{n}$ both percepts are fused into a single meaning. For $\mu>\mu_{\mathrm{n}}$ the stationary solution $\mathrm{v}^{*}(\mathrm{G})$ becomes multivalued. For maximum contrast $\mu=1$ the horizontal slope $(\mathrm{dG} / \mathrm{dv})^{-1}=0$ yields $\mathrm{v}_{\mathrm{i}}^{\infty}=2 \mathrm{i}-1, \mathrm{i}=1,2,3, \ldots$ as stable perception levels in the limit $\mathrm{G} \rightarrow \infty$.

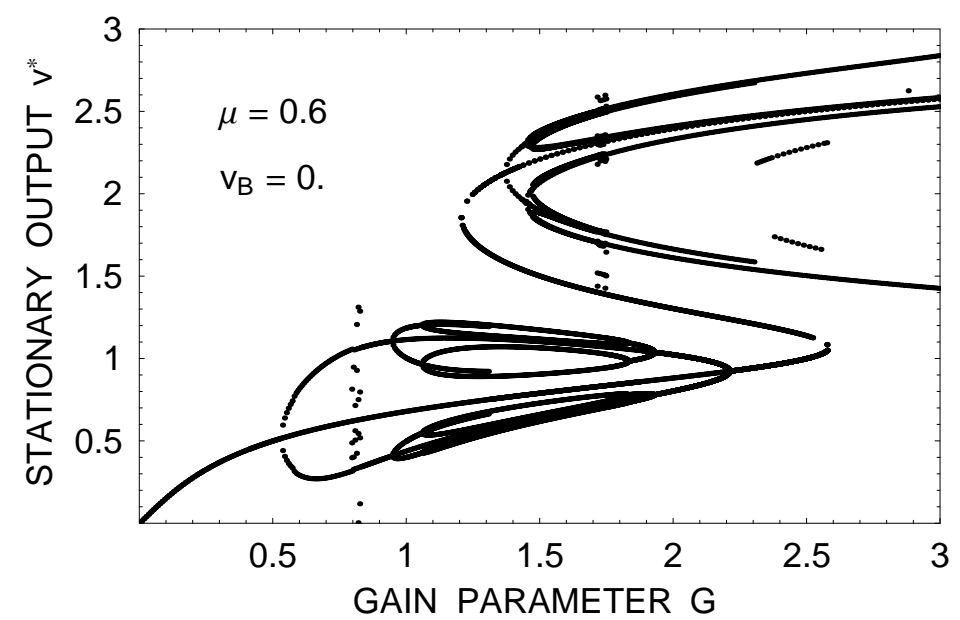

Fig. 3. Stationary solutions of equation (1) exhibiting $\mathrm{v}^{*}-$ hysteresis and period doubling bifurcations. Percept $1(\mathrm{P} 1)=$ lower positive slope $\mathrm{v}^{*}$-level; Percept $2(\mathrm{P} 2)=$ higher $\mathrm{v}^{*}$-level

Node bifurcation is required for explaining the existence of ambiguous perception within the present model. Under increasing stimulus strength I or feedback gain $g$ the stationary (1st order) perception state $\mathrm{v}^{*}$ jumps discontinuously from P1 to P2 at the turning points of the S-shaped hysteresis curve (= extrema of the inverse curve $\left.\mathrm{G}\left(\mathrm{v}^{*}\right)\right)$. The transition of $\mathrm{P} 2$ back to $\mathrm{P} 1$ occurs at a lower stimulus or gain parameter ( attention) value due to the hysteresis. The width of the instable negative slope section and the multivalued $\mathrm{G}$ - range is controlled by $\mu$. A similar hysteresis is observed for the coupling constants of columns of the visual cortex within the neuronal mean field theory [13].

A linear stability analysis of equation (1) yields the regions of instability, i.e. limit cycle and chaotic oscillations as dependent on the ratio $\tau / \mathrm{T}$ of damping time constant and feedback delay time [21][22]. Eigenfrequencies $\beta=2 \pi f$ are obtained via $\beta \tau=-\tan (\beta T)$. The analytical approximation for $\tau<<\mathrm{T}$ yields $\mathrm{f} \approx \mathrm{f}_{0} \mathrm{i} /(1-\tau / \mathrm{T}), \mathrm{i}=0$, $1,2, \ldots$ with $\mathrm{f}_{0}=1 / 2 \mathrm{~T}$, i.e. half of the inverse feedback delay time. With $\mathrm{T}=40 \mathrm{~ms}$ (delay between stimulus onset and V1 - response, see above) we obtain $\mathrm{f}_{0}=12.5 \mathrm{~Hz}$. With large damping time $\tau$ of the order of $\mathrm{T}$, period doubling oscillations are suppressed. 


\section{Computer Experiments}

\subsection{Simulated Perception - Attention Dynamics}

In this section I present numerical evaluations of the coupled differential - delay equations $(1,2)$ as obtained with the dynamical systems tool Matlab - Simulink (solver ode23tb for stiff problems). Figure 4 shows time series $v(t)$ and $G(t)$ for $I=1$, $\mu=0.6, T=2 T_{S}=40 \mathrm{~ms}$, time scale in units of the simulation interval $\mathrm{T}_{\mathrm{S}}, \tau / \mathrm{T}=0.1, \gamma$ $=60, \tau_{\mathrm{G}}=500$, attention bias $\mathrm{v}_{\mathrm{b}}=1.5$, noise variance $\mathrm{s}^{2}=0.001$, with stimulus - off sections $\left(\mu_{\mathrm{off}}=0.1, \mathrm{G}_{\mathrm{off}}=1.5\right)$ at the beginning and end of the time series.
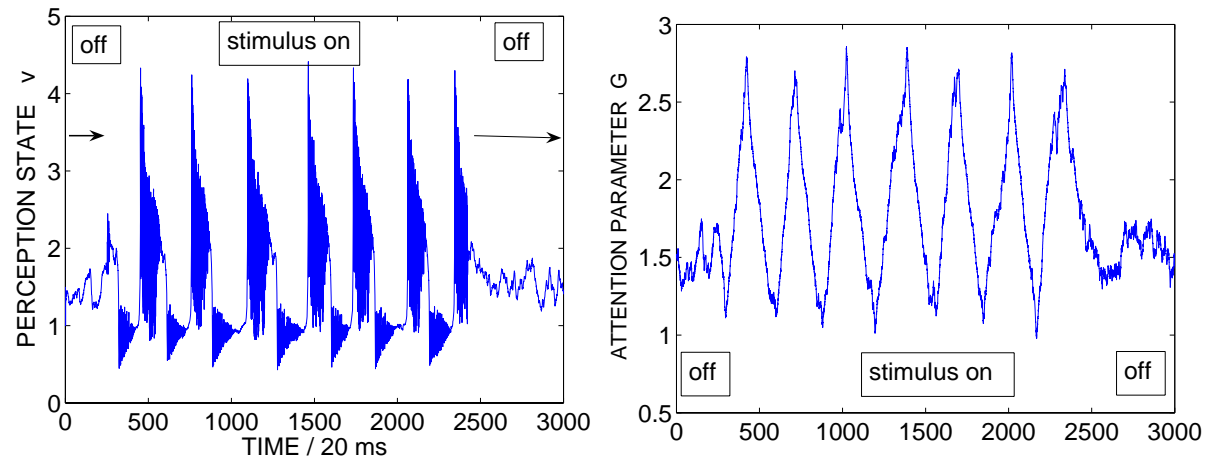

Fig. 4. Numerical evaluation of equations (1)(2) with stochastic noise variance $\mathrm{s}^{2}=0.001$. Left: perception state time series $v(t)$; right: attention parameter $G(t)$; Stimulus off $(\mu=0.1)$ during initial and final simulation phases. See text for simulation parameters

The time series of the perception state $\mathrm{v}(\mathrm{t})$ exhibits the spontaneous transitions between stationary perception states $\mathrm{P} 1$ (near $\mathrm{v}^{*} \approx 1$ ) and $\mathrm{P} 2$ (near $\mathrm{v}^{*} \approx 2.5$ ) with the expected superimposed limit cycle and chaotic oscillations. The transition time between P1 and P2 is of the order of $5-10 \mathrm{~T}_{\mathrm{S}} \approx 100-200 \mathrm{~ms}$, in reasonable agreement with the time interval between stimulus onset and conscious perception [11].

The phase space plot v vs. G in Fig. 5 exhibits separated regions of the stimulus off and stimulus - on (P1 and P2) states with trajectories of fast oscillations superimposed on the slow satiation (fatigue) dynamics.

The reversal time period is determined by the slow $\mathrm{G}(\mathrm{t})$ variation with satiation and recovery time constants $\gamma, \tau_{\mathrm{G}}$, with an absolute scale given by $\mathrm{T}=2 \mathrm{~T}_{\mathrm{S}}$. Limit cycle oscillations and deterministic chaos within P1, P2 is a characteristic of the individual perception states and has its origin in the finite delay time $\mathrm{T}$.

The effects of decreasing $\mathrm{T}$ and variation of attention bias $\mathrm{v}_{\mathrm{b}}$ are depicted in Figure 6 . The phase space trajectories in the left plot clearly show that with zero delay $(\mathrm{T}=$ 0 ) the limit cycle and chaotic oscillations vanish which are superimposed on the stationary perception states of the time series of Fig. 4 and the corresponding hysteresis loop in Fig. 5.

The $\mathrm{v}-\mathrm{G}$ - phase space plots exhibit a clear separation of stimulus - off $(\mu=0.1)$ and - on $(\mu=0.6)$ states due to the node bifurcation at $\mu=0.18$. The scattering of the 
reversal time period, however as indicated by the scattering of the P1 - P2 transitions, appears not to be significantly effected.

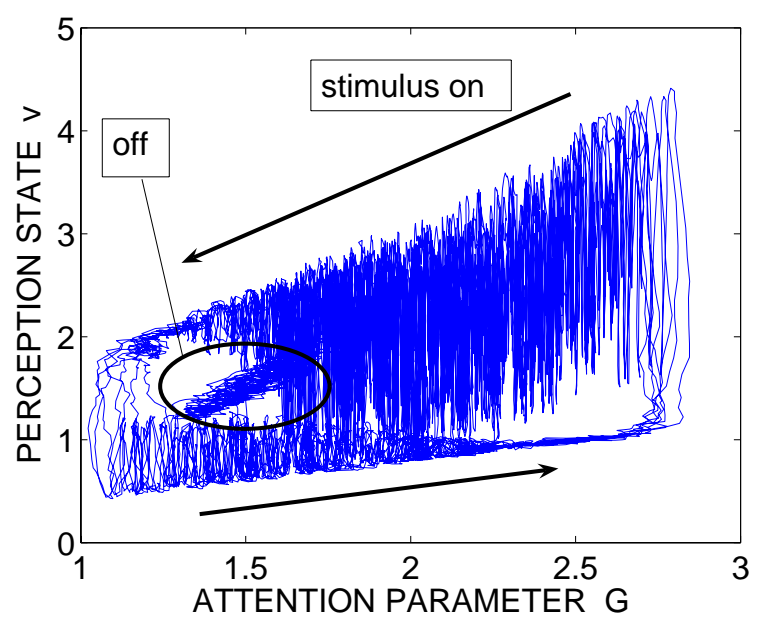

Fig. 5. Phase space trajectories v vs. G of the time series in Fig. 4 with the two control parameter values $\mu=0.1$ (=stimulus off) and $\mu=0.6$ (= stimulus on)

The right graph in Fig. 6 shows how the attention bias $\mathrm{v}_{\mathrm{b}}$ determines the relative dominance of one of the two percepts. In this example (noise variance $\mathrm{s}^{2}=0.001$ ), after stimulus on (percept 0 with $\mu=0.1$ is switched to $\mu=0.6$ ) the offset $\mathrm{v}_{\mathrm{b}}=0.9$ forces the perception to iterate to the lower perception state P1 with suppression of P2. Other parameters are the same as in Figure 5. $v_{b}$ may be used as a control parameter to model experimental results with perception biased towards one of the two percepts as reviewed in [3].
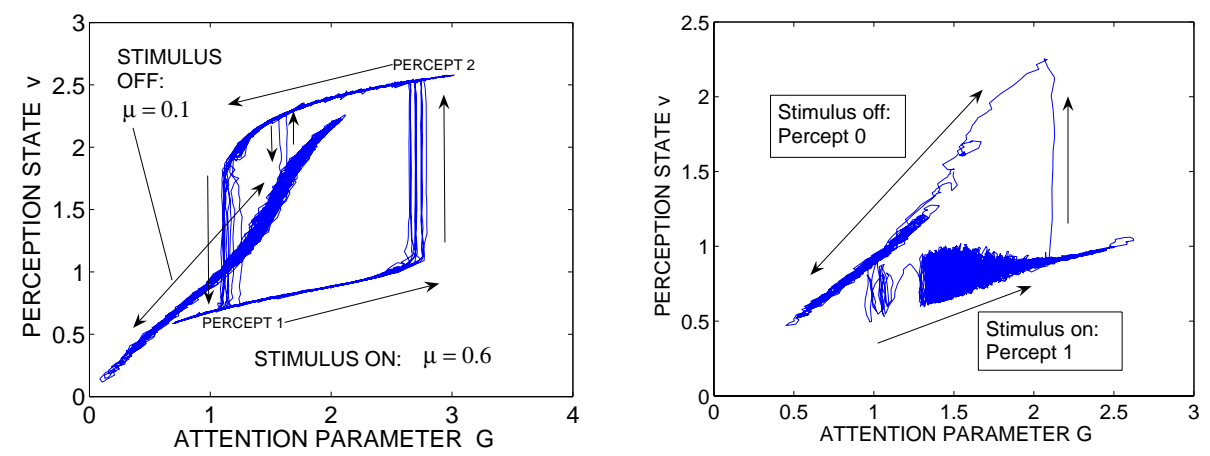

Fig. 6. Left: Perception - attention dynamics with zero feedback delay $(T=0)$ exhibiting vanishing limit cycle and chaotic oscillations. Phase space plot of perception - attention dynamics with attention bias $\mathrm{v}_{\mathrm{b}}=0.9$ and $\mathrm{T}=2$ 


\subsection{Reversal Time Statistics}

Figure 7 depicts the relative frequencies of the perceptual duration times of simulations as obtained by averaging 10 time series of $\mathrm{N}=50000$ iterations each, with $\mathrm{T}=$ $2, \tau=0.2, \gamma=60, \tau_{\mathrm{G}}=500, \mathrm{v}_{\mathrm{b}}=1.5$ and $\mathrm{s}^{2}=0.03$. Time series differ by noise (random number generator) seed value and perception state initial value $\mathrm{v}(\mathrm{t}=0)$.
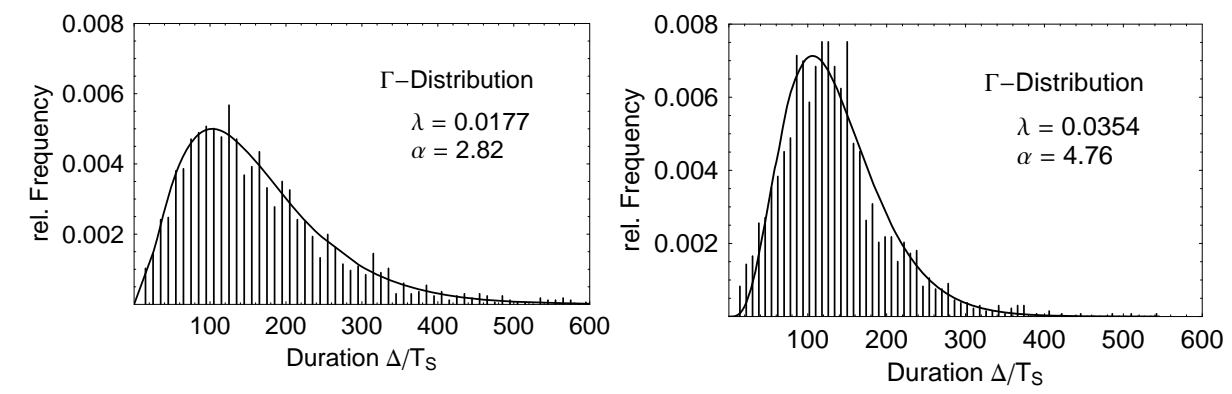

Fig. 7. Relative frequencies of perceptual duration time $\Delta$, in units $\mathrm{T}_{\mathrm{S}}=\mathrm{T} / 2$. Simulation parameters $\mu=0.6, \mathrm{v}_{\mathrm{b}}=1.5, \tau / \mathrm{T}=0.1, \gamma=60, \tau_{\mathrm{G}}=500, \mathrm{~s}^{2}=0.03$. Fit with $\Gamma$-distribution (solid line). Left: Percept P1 ( $\left.\mathrm{v}^{*} \approx 1\right)$, Right: Percept P2 $\left(\mathrm{v}^{*} \approx 2.5\right)$

Plotted are the two distributions of the perceptual durations $\Delta(\mathrm{P} 1)$ of percept 1 and $\Delta(\mathrm{P} 2)$ of percept 2. As suggested by a number of experimental results (e.g. [5][19] [27][29]) the relative frequencies are fitted by a $\Gamma$ - distribution as probability density with shape parameter $\alpha$ and scale parameter $\lambda$. Mean and variance are given by $\Delta_{\mathrm{m}}=$ $\alpha / \lambda$ and $\sigma^{2}=\alpha / \lambda^{2}$ respectively. For percept P1 and P2 mean and standard deviation are respectively $\Delta_{\mathrm{m}}=159 \mathrm{~T}_{\mathrm{S}}, \sigma=95 \mathrm{~T}_{\mathrm{S}}$ and $\Delta_{\mathrm{m}}=134 \mathrm{~T}_{\mathrm{S}}, \mathrm{s}=62 \mathrm{~T}_{\mathrm{S}}$. The ratios $\mathrm{s} / \Delta_{\mathrm{m}} \approx$ $0.4-0.6$ are in good agreement with the experimental findings reported in the literature.

\section{Discussion}

In contrast to [21] with simulation of a purely deterministic recursive process, and in agreement with [22] the addition of the stochastic attention noise $\mathrm{L}(\mathrm{t})$ leads to a significant increase of the variance, whereas the mean reversal times remain roughly the same as without noise, indicating the dominating influence of the deterministic dynamics on $\Delta_{\mathrm{m}}$. The limit cycle and chaotic contributions to the total variance in the present delay - differential model is significantly smaller as compared to the recursive approximation [22] without differential term. A dominating influence of stochastic processes was reported for binocular rivalry [7][28][29] where no significant chaotic contribution in the reversal time statistics was detected. It appears that with the given choice of model parameters the deterministic "noise" of the perception states P1, P2 is hardly detectible by analysis of reversal time measurements because the variance of the latter is dominated by the stochastic (attention) contribution. 
Table 1 lists results of $\Gamma$-distribution fits to the relative frequencies of perceptual duration times as obtained from computer simulations with delay times $\mathrm{T} / \mathrm{T}_{\mathrm{S}}=0,1,2$ (noise variance $\mathrm{s}^{2}=0.03$ ).

Table 1. Shape parameter, mean (in seconds), relative standard deviation and correlation coefficient for $\Gamma$-distribution fit to relative frequencies of perceptual duration times (separate for P1, P2) as obtained with different delay times

\begin{tabular}{|c|c|c|c|c|c|}
\hline & $\mathrm{T} / \mathrm{T}_{\mathrm{S}}$ & $\alpha$ & $\Delta_{\mathrm{m}} / \mathrm{s}$ & $\sigma / \Delta_{\mathrm{m}}$ & $\mathrm{R}^{2}$ \\
\hline P2 & 0 & 6.6 & 1.89 & 0.39 & 0.97 \\
\hline P2 & 1 & 7.5 & 2.62 & 0.37 & 0.98 \\
\hline P2 & 2 & 5.0 & 2.64 & 0.45 & 0.96 \\
\hline P1 & 0 & 3.5 & 2.69 & 0.53 & 0.95 \\
\hline P1 & 1 & 3.2 & 3.27 & 0.56 & 0.94 \\
\hline P1 & 2 & 2.5 & 3.22 & 0.63 & 0.93 \\
\hline
\end{tabular}

Fitting of the data by means of a $\Gamma$ - distributions for all $\mathrm{T}$ values yields correlation coefficients which show that only $3-7 \%$ of the total scattering is not explained by the $\Gamma$ - density function. The relative standard errors of the shape and scale parameters $\alpha$ and $\lambda$ respectively are around 3\% for all simulations. An important result is the fact that even with zero delay $(\mathrm{T}=0)$ the mean reversal time $\Delta_{\mathrm{m}}(\mathrm{T}=0)$ and the variance $\sigma^{2}$ is of the same order of magnitude as with finite delay. This indicates that the unavoidable contribution of the deterministic limit cycle oscillations and chaos (due to $\mathrm{T}>0$ for any realistic nonlinear physical system) to the reversal time variance is small as compared to the stochastic noise, in agreement with [28] and [10]. The shape parameter $\alpha$ is of interest with regard to the question if the dynamics is dominated by a Poisson process as suggested by Levelt [29]: in this case the $\alpha$ 's should cluster around natural numbers, a prediction which was verified in experiments by Murata et.al. [30].

\section{Conclusion}

A behavioral recursive nonlinear and stochastic phase oscillator model of spontaneous perceptual switching is presented which is related to previously published dynamical models [8][9]. The model is expected to help clarifying attention and perception related problems of augmented vision based human machine interfaces (e.g. [1][2]). The perception state is assumed to originate from interference between stimulus induced phase synchronized perception fields as proposed by the neuronal mean field theory [13], and it is coupled to the dynamics of an attention control parameter. Experimental results of Nakatani \& van Leeuwen [19] support the assumption that attentional effort which is expressed by eye blinking and saccade frequencies controls switching rates. By associating feedback delay time $\mathrm{T}$ with the stimulus onset - primary visual cortex (V1) response delay of $\sim 40 \mathrm{~ms}$ [11] absolute values of mean perceptual duration times of $\Delta_{\mathrm{m}} \approx 3 \mathrm{~s}$ are obtained, in reasonable agreement with published experimental results $(1-10 \mathrm{~s}$, e.g. [5][27]). The large inter - subject variations 
of $\Delta_{\mathrm{m}}$ can be modeled by suitable choice of contrast (difference of meaning) parameter $\mu$, satiation (fatigue) and recovery time constants $\gamma$ and $\tau_{\mathrm{G}}$ respectively, and noise variance $s^{2}$. The relative duration of dominance vs. suppression times is determined by the attention bias parameter $\mathrm{v}_{\mathrm{b}}$. The magnitude of limit cycle and chaotic oscillations with eigenfrequencies $<100 \mathrm{~Hz}$ is controlled by the ratio $\tau / \mathrm{T}$ of perceptual damping time constant and delay time. Because the reversal time statistics is only weakly dependent on $\mathrm{T}$ it is concluded that the limit cycle and chaotic oscillations which are superimposed on the stationary perception states, also contribute only weakly to the reversal time statistics, in agreement with results of other authors [28][10]. The present model thus supports the proposal of Poston \& Stewart [20] of a deterministic catastrophe topology as the basis of the perception reversal dynamics, with the higher moments of the statistics determined by a stochastic process which in certain cases (binocular rivalry) hides the deterministic contribution. Ongoing work aims at quantifying the amount of long range correlations of the time series by evaluating the Hurst parameter as proposed by Gao et.al.[31] and the reproduction of the proposed Poisson process [29][30] superimposed on the deterministic dynamics by generating a sufficient statistical basis of shape parameters $\alpha$ of the $\Gamma$-distributions.

\section{Acknowledgement}

I am indebted to Monika Mittendorf for help in writing the Mathematica and Matlab code of the numerical simulation and analysis software and in performing the statistical analysis. I would like to thank H. Nakatani of Riken Brain Science Institute for information on some recent experimental results.

\section{References}

1. Peli, E.: Visual issues in the use of a head-mounted monocular display. Optical Engineering 29 (1990) 883-892

2. Laramee, R.S., Ware, C.: Rivalry and Interference with a Head Mounted Display. ACM Transactions on Computer-Human Interactions 9, (2002) 238-251

3. Engel, A.K., Fries, P., König, P., Brecht, M., Singer, W.: Temporal binding, binocular rivalry, and consciousness. Consciousness and Cognition 8 (1999) 128-151

4 Orbach, J., Ehrlich, D., Heath, H.A: Reversibility of the Necker Cube: An examination of the concept of satiation of orientation, Perceptual and Motor Skills 17 (1963) 439-458

5 Borsellino, A., de Marco, A., Allazetta, A., Rinesi, S., Bartolini, B.: Reversal time distribution in the perception of visual ambiguous stimuli. Kybernetik 10 (1972) 139-144

6. Hupe, J.-M. , Rubin N.: The dynamics of bistable alternation in ambiguous motion displays: a fresh look at plaids, Vision Research 43, (2003) 531-548

7 Richards, W., Wilson, H.R., Sommer, M.A.: Chaos in percepts. Biol. Cybern. 70 (1994) 345-349

8. Ditzinger T., Haken H.: A Synergetic Model of Multistability in Perception. In: Kruse, P., Stadler, M. (eds.): Ambiguity in Mind and Nature. Springer-Verlag, Berlin: (1995) 255273. 
9. Kelso J.A.S., Case P., Holroyd T., Horvath E., Raczaszek J., Tuller B., Ding M.. Multistability and metastability in perceptual and brain dynamics. In: Kruse, P., Stadler, M. (eds.): Ambiguity in Mind and Nature. Springer-Verlag, Berlin: (1995) pp. 255-273

10. Merk, I. L. K., Schnakenberg, J.: A stochastic model of multistable perception. Biol.Cybern. 86, (2002) 111-116

11. Lamme, V.A.F.: Why visual attention and awareness are different. Trends in Cognitive Sciences , (2003) 12-18

12. Tononi, G. , Edelman, G.M.: Consciousness and Complexity. Science 282 (1998) 18461851

13. Schuster, H.G., Wagner, P.A.: A Model for Neural Oscillations in the Visual Cortex: 1. Mean field theory and the derivation of the phase equations. Biol. Cybernetics 64 (1990) 7782

14. Blake, R., Logothetis, N.K.: Visual competition. Nature Reviews / Neuroscience 3 (2002) $1-11$

15. Engel, A.K., Fries, P., Singer, W.: Dynamic Predictions: Oscillations and Synchrony in Top-Down Processing. Nature Reviews Neuroscience, 2 (2001) 704-718

16. Engel, A.K., Fries, P., König, P., Brecht, M., Singer, W.: Temporal binding, binocular rivalry, and consciousness. Consciousness and Cognition 8 (1999) 128-151

17. deGuzman, G. C., Kelso, J. A. S.: Multifrequency behavioral patterns and the phase attractive circle map. Biological Cybernetics 64 (1991) 485-495

18. Srinavasan, R., Russel, D.S., Edelman, G M, Tononi, G.: Increased synchronization of magnetic responses during conscious perception, J. Neuroscience 19 (1999) 5435 - 5448

19. Nakatani H., van Leeuwen C.: Individual differences in perceptual switching rates; the role of occipital alpha and frontal theta band activity. Biol. Cybern. 93 (2005) 343-354

20. Poston T., Stewart, I.: Nonlinear Modeling of Multistable Perception. Behavioral Science 23, (1978) 318-334

21. Fürstenau N.: Nonlinear dynamics model of cognitive multistability and binocular rivalry, Proceedings IEEE 2003 Int. Conf. on Systems, Man and Cybernetics, IEEE cat. no. 03CH37483C (2003) 1081-1088

22. Fürstenau N.: A chaotic attractor model of cognitive multistability. Proceedings IEEE Int. Conf. on Systems, Man and Cybernetics, IEEE cat. no. 04CH37583C (2004) 853- 859

23. Watts, C., Fürstenau, N.: Multistable fiber-optic Michelson Interferometer exhibiting 95 stable states. IEEE J. Quantum Electron 25 (1989) 1-5

24. Fürstenau, N.: Bistable fiber-optic Michelson interferometer that uses wavelength control. Optics Letters 16 (1991) 1896-1898

25. Edelman, G.: Wider than the Sky. Penguin Books (2004) pp. 87-96

26. Hillyard, S.A., Vogel, E.K. Luck, S.J.: Sensory gain control (amplification) as a mechanism of selective attention: electrophysiological and neuroimaging evidence. In: Humphreys, G.W. Duncan, J.\& Treisman, A. (eds.): Attention, Space, and Action. Oxford University Press (1999) 31-53

27. Zhou, Y.H., Gao, J.B., White, K.D., Merk, I., Yao, K.: Perceptual dominance time distributions in multistable visual perception. Biol. Cybern. 90 (2004) 256-263

28. Lehky S. R.: Binocular rivalry is not chaotic, Proc. R. Soc. Lond. B 259 (1995) 71-76

29. Levelt, W.J.M.: Note on the distribution of dominance times in binocular rivalry. Br. J. Psychol. 58 (1967) 143-145

30. Murata, T., Matsui, N., Miyauchi. S., Kakita, Y., Yanagida, T.: Discrete stochastic process underlying perceptual rivalry. NeuroReport 14 (2003) 1347-1352

31. Gao, J.B., Merk, I., Tung W W, Billok V, White, K.D., Harris J G, Roychowdhury V P.: Inertia and memory in ambiguous visual perception. preprint Phys. Rev. Lett., (2005) submitted 